\title{
Revue Revue de l'histoire des religions del'histoire des religions

The Greek Life of St. Leo bishop of Catania (BHG 981b), Text and Notes by Alexandros G. ALEXAKIS, Translation by Susan WESSEL

Bruxelles, Société des Bollandistes (« Subsidia hagiographica », 91), 2011, XXXVII-355 p., 25 cm, ISBN 978-2-87365-026-1.

\section{Annick Peters-Custot}

\section{CpenEdition}

\section{Journals}

Édition électronique

URL : http://journals.openedition.org/rhr/8278

DOI : $10.4000 /$ rhr.8278

ISSN : 2105-2573

Éditeur

Armand Colin

\section{Édition imprimée}

Date de publication : 1 octobre 2014

Pagination : 504-507

ISBN : 978-2-200-92912-1

ISSN : 0035-1423

Référence électronique

Annick Peters-Custot, « The Greek Life of St. Leo bishop of Catania (BHG 981b), Text and Notes by Alexandros G. ALEXAkIS, Translation by Susan weSSEL », Revue de l'histoire des religions [En ligne], 3 | 2014, mis en ligne le 03 novembre 2014, consulté le 22 septembre 2020. URL : http:// journals.openedition.org/rhr/8278; DOI : https://doi.org/10.4000/rhr.8278

Ce document a été généré automatiquement le 22 septembre 2020.

Tous droits réservés 


\section{The Greek Life of St. Leo bishop of Catania (BHG 981b), Text and Notes by Alexandros G. ALEXAKIS, Translation by Susan WESSEL}

Bruxelles, Société des Bollandistes (« Subsidia hagiographica », 91), 2011, XXXVII-355 p., 25 cm, ISBN 978-2-87365-026-1.

\section{Annick Peters-Custot}

\section{RÉFÉRENCE}

The Greek Life of St. Leo bishop of Catania (BHG 981b), Text and Notes by Alexandros G. ALEXAKIS, Translation by Susan WESSEL, Bruxelles, Société des Bollandistes (« Subsidia hagiographica », 91), 2011, XXXVII-355 p., 25 cm, ISBN 978-2-87365-026-1.

1 Le dossier hagiographique de Léon, évêque de Catane en Sicile, est un des plus originaux et des plus complexes de l'hagiographie médiévale en langue grecque. Logiquement, les débats savants qui entourent le dossier sont intenses et stimulés par chaque nouvelle publication. Toutefois, l'intérêt du dossier hagiographique de Léon dépasse largement le cercle restreint des spécialistes de l'hagiographie iconoclaste, crypto-iconoclaste et iconodoule, tant le contenu de cette Vie et les mystères de son élaboration peuvent susciter d'intérêt pour tout historien curieux des querelles internes à l'Église comme des procédés d'élaboration littéraire de la doxa religieuse, politique et littéraire consécutive à la domination finale d'une des parties.

2 La Vie de saint Léon, natif de Ravenne, puis évêque de Catane, est caractérisée par un déséquilibre étonnant, au sens où le saint n'apparaît que comme un protagoniste secondaire de la narration, dont l'essentiel des chapitres est consacré à son adversaire, un anti-héros peu commun, le mage Héliodore, puissante figure du mal et préfiguration de Faust. En effet, après quelques chapitres peu originaux sur la vie de Léon, le discours 
hagiographique transfère l'intérêt du lecteur sur l'objet qui fournira l'occasion du plus magnifique des - peu nombreux - miracles de Léon : Héliodore. Ce dernier obtient par l'entremise d'un magicien juif d'entrer en contact avec le diable. Reniant le Sauveur, il reçoit l'aide d'un démon, Gaspar, qui le fera bénéficier de sa puissance maléfique. À partir de là, la Vie de Léon décrit les méfaits d'Héliodore à Catane comme à Constantinople, où il est par deux fois convoqué par les empereurs. Transformant les pierres en or et inversement, abusant de la naïveté de la population, qu'il engage vers des pratiques idolâtres, impliquant même le neveu de l'évêque Léon dans une affaire de cheval magique, Héliodore met à mal l'ordre religieux, moral et économique de la cité. Enfin, provoquant l'évêque par ses blasphèmes, Héliodore est entraîné par et avec le saint prélat sur un bûcher qui réduit le mage en cendres, tandis que l'évêque sort indemne.

Le dossier hagiographique de Léon comprend plusieurs versions en grec, parmi lesquelles deux témoins centraux, appelés version A (ou VLA) et version B (ou VLB), cette dernière faisant l'objet du présent ouvrage ; une vie métrique du XIII ${ }^{\mathrm{e}}$ siècle, qui est une réécriture plus littéraire que d'édification; des hymnes de Joseph l'Hymnographe. La Vie de Léon est également connue par une version latine, assez verbeuse, réélaboration d'une Vie grecque perdue. Les débats les plus intenses portent surtout sur les deux Vies grecques, VLA et VLB car c'est sur les relations, les datations et les contextes d'écriture respectifs de ces deux Vies que repose l'essentiel des débats sur l'identification des principaux protagonistes - Héliodore, Léon et les empereurs de Constantinople - et sur l'idéologie qui présida à la rédaction de ces documents. En effet, ce dossier est à ce point complexe que les spécialistes débattent encore de savoir si certaines de ses pièces sont de facture originelle iconoclaste, ou d'emblée dévouées à la cause iconophile. Le présent ouvrage s'inscrit donc dans une série de débats parfois polémiques, dont il prend sa part, au milieu de grands noms de l'histoire, de la littérature et de l'hagiographie byzantine des époques iconoclaste et post-iconoclaste : I. Ševčenko, M.-F. Auzépy, A. Acconcia Longo, I. Rochow, etc.

4 VLA (BHG 981) a été publiée par A. Acconcia Longo à partir des manuscrits connus, tous italo-grecs. Elle est de facture plus fruste que VLB (BHG 981b), publiée en 1914 par Latišev à partir d'un manuscrit unique, et rééditée ici à partir de sept des neuf manuscrits connus. Il faut souligner ici l'ampleur du travail mené par l'A. (la traduction étant due à S. Wessel) : l'ouvrage propose, après une imposante bibliographie, une copieuse introduction qui précède l'édition du texte avec traduction anglaise en regard, une série de commentaires brefs et ponctuels chapitre par chapitre, en appendice, l'édition et la traduction anglaise de la Laudatio Leonis Ep. Cataniae (BHG 981d), enfin de nombreux et fort utiles indices. L'ensemble est impressionnant et justifie pleinement les années que l'A. a passées sur ce dossier majeur. Il est certain que c'est l'ample introduction, qui détaille minutieusement le dossier hagiographique (état de la recherche et interprétations successives, datation de VLB, auteur, composition, niveau rhétorique du texte, identification des personnages, tradition manuscrite) qui peut susciter le plus de commentaires, en particulier en ce qu'il s'oppose frontalement aux interprétations les plus récentes (celles d'Acconcia Longo). Les points d'accord entre les deux auteurs ne sont pas nuls: le héros, Léon, serait un saint factice; VLB, contrairement à $V L A$, aurait été écrite loin de la Sicile - le texte atteste une méconnaissance substantielle de la géographie de lîle. Enfin, l'auteur de VLB paraît être un lecteur assidu et un utilisateur habile des traités de rhétorique grecque, ce que 
démontre l'A. dans son étude, confirmant les caractéristiques habituelles de la littérature byzantine de haut niveau.

5 Exposons brièvement les antagonismes: Acconcia Longo estime que VLA constitue une des réécritures, probablement la plus ancienne, d'un texte original iconoclaste par la suite censuré, mais transmis par versions remaniées. La réécriture, probablement sicilienne, de VLA est maladroite, ce qui lui permet de transmettre, presque à son insu, des éléments du texte original, notamment dans son préambule, dans lequel Acconcia Longo voit une version de bas niveau de l'Horos, la définition de foi, du concile de Hiereia (754) organisé par Constantin V pour affirmer la doctrine iconoclaste. Pour Acconcia Longo, VLB relèverait d'une autre branche des versions remaniées de l'original iconoclaste censuré, une version très littéraire et postérieure à l'affirmation de l'orthodoxie iconophile. L'A. du présent ouvrage présente un tout autre tableau. Selon lui, la première des Vies de Léon serait VLB, une hagiographie iconodoule rédigée en plein second iconoclasme, donc par un auteur qui risquait sa vie à valoriser un prélat iconophile. La facture du texte incite l'A. à identifier l'auteur de VLB avec un membre du cercle de Michel le Syncelle. VLA, postérieure, constituerait une autre version, également iconophile, de la vie de Léon. Enfin, l'A. identifie Héliodore, le mage sulfureux, avec le patriarche iconoclaste Jean VII le Grammairien, ce qui l'incite à voir dans Léon une représentation littéraire du patriarche adverse, Nicéphore.

Dans l'économie de la démonstration proposée par l'A., certains éléments argumentatifs ont paru peu probants. Que le préambule de VLB évoque une commande à l'origine de l'écriture, atteste-t-il forcément que VLB est la première mise par écrit de la vie de Léon (p. 34) ? Que la légende d'Empédocle, en préambule de VLB, n'apparaisse pas dans VLA est-il vraiment la preuve d'une réécriture sicilienne de VLA (ibid.) ? Cela semble peu convaincant. Or, on l'a vu, c'est sur la datation relative de VLA et de VLB que repose une bonne part de l'argumentation sur l'idéologie sous-jacente aux textes. Soulignons, en passant, combien il paraît peu explicable qu'un auteur lettré de la première moitié du IX ${ }^{e}$ siècle (selon la date proposée de VLB par l'A.) méconnaisse la Sicile - alors stratégique pour l'Empire d'Orient - au point de confondre Palerme (dont l'importance date de l'époque de la Sicile islamique) avec Syracuse, principale ville sicilienne à l'époque byzantine.

7 Surtout, il semble que l'A. fait un usage récurrent, dans l'économie de la preuve, de topoi hagiographiques, alors que l'état de la documentation ne permet pas de savoir s'ils constituaient un apanage iconoclaste, iconodoule ou partagé par les deux partis. En effet, la disparition de la plupart des sources iconoclastes et les réécritures subies par les autres, du fait de la censure iconophile, génèrent des incertitudes profondes quand il s'agit de déterminer si telle comparaison, modalité d'expression, topos hagiographique ou procédé rhétorique, ou telle accusation d'hérésie est le fait plutôt d'un parti que de l'autre. Il est probable que les auteurs de l'un ou de l'autre bord partageaient des origines sociales proches, la même éducation patristique, littéraire et philosophique, se nourrissaient des mêmes exemples bibliques, historiques ou mythologiques et des mêmes traités de rhétoriques, et donc truffaient leurs écrits de références, accusations, et procédés identiques. La présence de tels éléments ne saurait donc être probante dans l'appréhension de l'idéologie sous-jacente. Si l'iconoclaste Jean VII le Grammairien fut comparé par la rhétorique iconodoule à Simon le Magicien, cette identification, liée à l'accusation de magie, a touché des individus hors du contexte du conflit iconoclaste. L'antijudaïsme - illustré, dans VLB, par l'aide apportée 
par le magicien juif à Héliodore - fut également un élément partagé par les partis iconoclaste et iconodoule. On peut émettre semblable réserve sur bien des arguments proposés par l'A.

8 Ainsi, l'A. procède souvent par accumulation d'éléments en eux-mêmes peu probants afin de démontrer sa thèse. Il a l'honnêteté de souligner lui-même la faiblesse de certains arguments, voire de proposer des interprétations divergentes des siennes. On a ainsi apprécié la note 157 de la p. 73, qui évoque une datation de VLB postérieure, et hors contexte iconoclaste, à celle que l'A. préfère. Cette honnêteté, alliée à l'intérêt de l'entreprise de réédition, à une érudition solide, au caractère minutieux de l'argumentation et de l'analyse socio-littéraire du texte, constituent les éléments forts d'un ouvrage qui prend le risque d'être critiqué.

\section{AUTEURS}

\section{ANNICK PETERS-CUSTOT}

Université de Saint-Étienne. 